Article

\title{
Comparative Analysis of Reliability Indices and Hydraulic Measures for Water Distribution Network Performance Evaluation
}

\author{
Gimoon Jeong and Doosun Kang * \\ Department of Civil Engineering, Kyung Hee University, 1732 Deogyeong-daero, Giheung-gu, Yongin-si, \\ Gyeonggi-do 17104, Korea; gimoon1118@gmail.com \\ * Correspondence: doosunkang@khu.ac.kr
}

Received: 5 August 2020; Accepted: 25 August 2020; Published: 26 August 2020

\begin{abstract}
The performance of water distribution networks (WDNs) can be quantified by several types of hydraulic measure. In design and operation of a WDN, sufficient consideration should be given to system performance, and it would be inefficient to separately consider individual characteristics of hydraulic measures. Instead, various reliability indices have been developed and utilized to evaluate the performance of WDNs; however, deciding which index to use according to a particular WDN situation has not been investigated in sufficient depth. In this regard, this study analyzes the correlation between representative reliability indices and hydraulic measures to propose the most adequate reliability index according to the desired system performance in various situations. Specifically, six hydraulic measures representing system performance were selected from the viewpoint of redundancy, robustness, and serviceability. In addition, nine indices for estimating system reliability were classified based on theoretical backgrounds such as hydraulic, topological, entropic, and mixed approaches. The correlations between the nine indices and six measures were analyzed using 17 sample hypothetical networks with different layouts, under three water supply scenarios, and the overall evaluation results for each reliability index are presented through multi-criteria decision analysis.
\end{abstract}

Keywords: comparative analysis; hydraulic measure; multi-criteria decision analysis (MCDA); reliability index; water distribution network (WDN)

\section{Introduction}

The expected performance of an infrastructure system can be interpreted through the concept of "system reliability", which quantifies marginal capacity to fulfil the users' requirements. In a water distribution network (WDN), the system reliability indicates the stable performance of supplying required water with adequate service pressure. Here, the specific performance of WDN could be assessed by representative hydraulic measures.

Wildavsky [1] defined "resilience", one of the most important performance parameters of WDNs, as the capacity to cope with unanticipated dangers after they have become manifest and learning to bounce back. Subsequently, Comfort [2] defined resilience as the capacity to adapt existing resources and skills to new situations and operating conditions. For theoretical concepts of reliability, Maier et al. [3] suggested first-order estimators such as reliability, vulnerability, and resilience of water quality service in rivers, and Bruneau et al. [4] summarized the seismic resilience of an infrastructure system into $4 \mathrm{R}$ 's: robustness, redundancy, resourcefulness, and rapidity. For reliability assessment of WDNs, several studies [5-7] compared the performance of different WDNs using simple types of representative hydraulic measure such as average surplus head, minimum surplus head, and supplied demand. Moreover, Marlim et al. [8] divided and formulated the reliability objectives of a WDN's 
user service into social, economic, hydraulic, and water quality, and Markov et al. [9] also found that the performance of a WDN could be measured via users' satisfaction and proposed a serviceability indicator to quantify this.

However, any individual hydraulic measurement is too fragmentary to be applied as the objective for WDN design and operation; hence, a large number of studies have been attempting to formulate a single "synthetic" index using various theoretical approaches. Wagner et al. [10] were the first to introduce and apply the concepts of mechanical and hydraulic reliability approaches to WDNs. Mays [11] also defined mechanical reliability as network topology evaluating system connectivity, given failure conditions, and hydraulic reliability as the ability of a system to meet the required water demand and pressure under normal and abnormal conditions. Later, Ostfeld [12] categorized WDN reliability evaluations into topological, hydraulic, and entropic backgrounds.

With regard to the hydraulic approach, Todini [13] developed the resilience index (RI), which represents the surplus and required energy in a WDN, whereas Jayaram and Srinivasan [14] developed a modified resilience index (MRI) with a different energy composition. Later, Liu et al. [15] and Jeong et al. [16] identified that a topographical relationship alters the reliability of the network performance, and proposed mixed reliability indices, a pipe hydraulic resilience index (PHRI) and a revised resilience index (RRI) by incorporating hydraulic and topographical approaches.

Within topological methods, research using a geometric approach [17-19] was performed, leading to different measures for estimating network reliability such as network efficiency (NE), average degree (AD), and link density. Creaco et al. [7] found that network performance is represented by the uniformity of pipe diameters in loop structures and developed a uniformity coefficient as the topological index. Moreover, Prasad and Park [6] also proposed a mixed reliability index, namely network resilience index (NRI), considering diameter uniformity along with the existing resilience index.

Regarding entropic reliability approaches, Awumah et al. [20] proposed an entropy reliability index by formulating water supply diversity in a WDN, and Tanyimboh and Templeman [21] developed and applied flow entropy (FE) into a WDN study based on the entropy concept of Shannon [22]. Raad et al. [23] suggested another mixed reliability index incorporating hydraulic and entropic approaches and compared four different reliability indices using performance measures in a benchmark network. Moreover, Jeong and Kang [24] suggested a hydraulic uniformity index (HUI), which is a mixed reliability index considering uniformity of the hydraulic gradients of pipes within a WDN.

However, the previously mentioned reliability indices have a bias towards certain system performances as influenced by their theoretical background. For example, in a recent study by Paez et al. [25], the correlation between different indices was analyzed through five arbitrary network designs. In addition, Tanyimboh et al. [26] investigated the correlations between surrogate reliability/redundancy measures (e.g., FE, RI, NRI) and surplus power factor with hydraulic reliability in hypothetical WDNs. In the most recent study of Sirsant and Reddy [27], the correlation between a reliability index and hydraulic and mechanical performance was also analyzed based on an optimally designed network and multipurpose functions of design cost, entropy, resiliency, and combined indices.

Eventually, it is necessary to appropriately examine which reliability index best reflects each type of system performance according to various situations and purposes required in the design and operation of WDNs. To that end, in this study, the correlations between representative reliability indices and hydraulic measures under three abnormal conditions (pipe failure; demand increase; fire flow) are analyzed for various types of application networks. Through these simulations, it was intended to determine the most adequate reliability index for evaluation of WDN performance in various abnormal water supply conditions.

The rest of this paper is organized as follows. The following section provides an overview of the proposed correlation analysis, and details of the hydraulic measures and various reliability indices are also described. Section 3 explains the design process of application networks and three application scenarios, and the application results and analyses are provided in Section 4. Finally, the conclusions of the study are summarized in Section 5. 


\section{Methodology}

\subsection{Overview of Correlation Analysis}

In this study, representative reliability indices and hydraulic measures were classified and estimated for application networks following the procedure shown in Figure 1. First, nine reliability indices are selected and estimated through a base scenario representing normal operating conditions. Meanwhile, hydraulic measures show the change in performance of the system under abnormal conditions (as compared with normal conditions), and thus six hydraulic measures according to performance properties were selected and simulations were performed according to three scenarios representing "abnormal" conditions. The overall framework can be summarized as follows: (1) categorization of hydraulic measures and reliability indices, (2) establishing synthetic application networks, (3) calculation of the measures and indices in normal/abnormal water supply conditions for each application network, (4) correlation analysis between the measures and indices, and (5) multi-criteria decision analysis (MCDA) for evaluation of reliability indices.

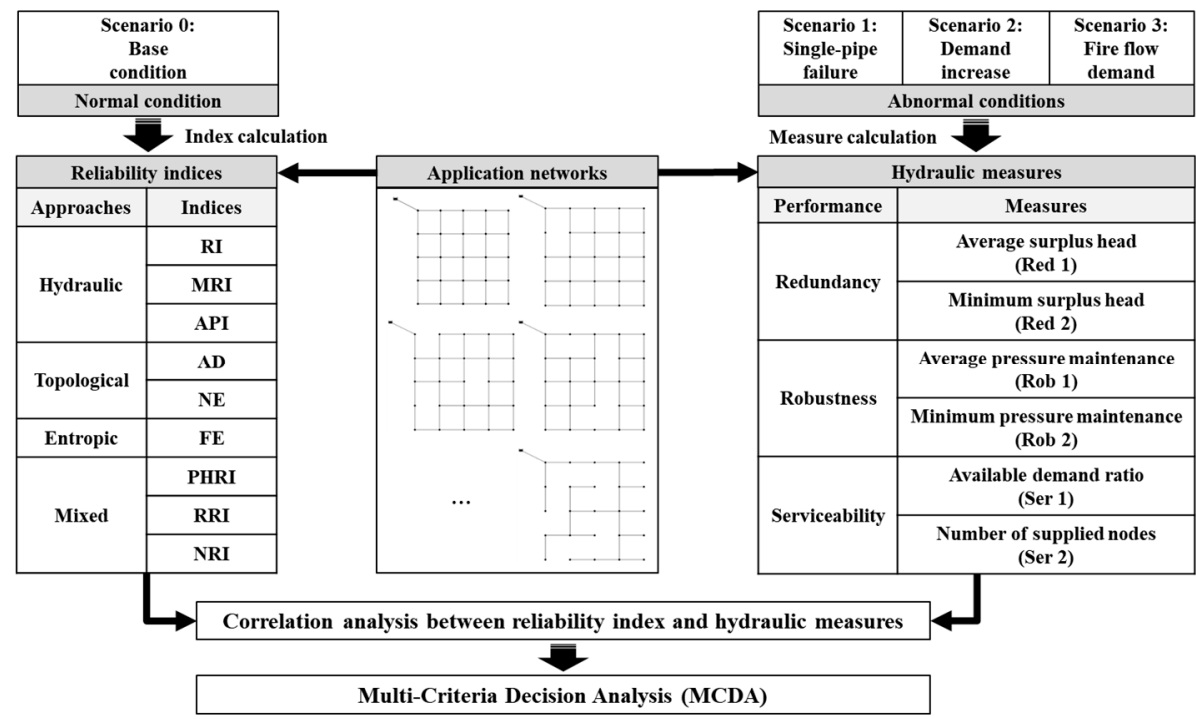

Figure 1. Correlation analysis between reliability indices and hydraulic measures. RI: resilience index, MRI: modified resilience index, API: available power index, AD: average degree, NE: network efficiency, FE: flow entropy, PHRI: pipe hydraulic resilience index, RRI: revised resilience index, NRI: network resilience index.

\subsection{Hydraulic Measures}

Hydraulic measures are an indication of the water supply's status in the system, which directly represents the performance of a WDN. In this study, the properties that can ensure the performance of a WDN were classified as redundancy, robustness, and serviceability, and change in performance was quantified based on two hydraulic measures of each property. Detailed descriptions of the concept of each performance property and the selected hydraulic measures are presented below.

\subsubsection{Redundancy Measures}

Redundancy used to be defined as substitutable capacity such as excessive backup [4] and the extent captured by the loops [28] in WDN design. In this study, network average surplus head (Red1) and minimum surplus head (Red2) under abnormal conditions were used as measures to indicate the redundancy of the system. Here, the surplus head refers to the head supplied in excess of the required head at each node (i.e., total head, subtracting elevation and minimum required pressure 
head); it can be shown that the higher the values of Red1 and Red2, the higher the network redundancy. The detailed calculation of redundancy measures can be presented as follows:

$$
\begin{gathered}
\operatorname{Red} 1=\frac{\sum_{j=1}^{\text {nnode }}\left(H_{j}-H_{\text {req }, j}\right)}{\text { nnode }} \\
\operatorname{Red} 2=\min \left\{H_{j}-H_{\text {req }, j}\right\}, j=1,2, \ldots, \text { nnode }
\end{gathered}
$$

where nnode is the number of nodes; $H_{j}$ is the total head at node $j$; and $H_{\text {req, }}$ is the minimum required head at node $j$.

\subsubsection{Robustness Measures}

Robustness represents the capacity of a system to withstand a given level of stress or demand without suffering degradation or loss of function [4]. Hence, robustness performance keeps the variability of losses within a narrow band [29]. In this study, network average pressure maintenance (Rob1) and minimum pressure maintenance (Rob2) under abnormal conditions (as opposed to normal conditions) were used as measures to reflect the robustness of the system. Herein, pressure maintenance refers to the ratio between nodal pressure under abnormal and normal conditions (i.e., pressure under abnormal conditions divided by the pressure under normal conditions), and it can be understood that the higher the values of Rob1 and Rob2, the stronger the robustness of the network. The detailed calculation of robustness measures can be expressed as follows:

$$
\begin{gathered}
\text { Rob1 }=\frac{1}{\text { nnode }} \sum_{j=1}^{\text {nnode }} \frac{H_{j, \text { abnormal }}}{H_{j, \text { normal }}} \\
\text { Rob2 }=\min \left\{\frac{H_{j, \text { abnormal }}}{H_{j, \text { normal }}}\right\}, j=1,2, \ldots, \text { nnode }
\end{gathered}
$$

where $H_{j, n o r m a l}$ and $H_{j, a b n o r m a l}$ are the total heads at node $j$ in normal and abnormal water supply conditions, respectively.

\subsubsection{Serviceability Measures}

Serviceability represents users' satisfaction with a system's functionality, depending on several factors such as the vulnerability of system components, topology, and operation scenarios [9]. In this study, the available demand ratio (Ser1) and number of supplied nodes (Ser2) under abnormal conditions were used as measures to represent the serviceability of the system. Herein, the available demand ratio indicates the ratio between the nodal supplied demand under abnormal and normal conditions (i.e., supplied demand under abnormal conditions divided by the desired demand), and supplied node refers to a node in which all desired demands are satisfied. Therefore, it can be understood that the higher the values of Ser1 and Ser2, the better the serviceability of the network. The detailed calculation of serviceability measures can be presented as follows:

$$
\begin{gathered}
\text { Ser1 }=\frac{\sum_{j=1}^{\text {nnode }} Q_{j, \text { avl }}}{\sum_{j=1}^{\text {nnode }} Q_{j}} \\
\text { Ser2 }=\sum_{j=1}^{\text {nnode }} A_{j} \text { where } A_{j}=\left\{\begin{array}{cc}
1 & \text { if } Q_{j, \text { avl }}=Q_{j} \\
0 & \text { otherwise }
\end{array}\right.
\end{gathered}
$$

where $Q_{j}$ is the water demand at node $j ; Q_{j, a v l}$ is the supplied water demand at node $j$; and $A_{j}$ is the water availability indicator of node $j$. 


\subsection{Reliability Assessment Index}

A reliability index can be defined as an indicator calculated by system states such as elements, measurements, and structural characteristics. So far, various indices have been proposed through hydraulic, topological, and entropic approaches in search of a comprehensive understanding of the reliability of a system, and furthermore, these indices have been combined with each other or developed in numerous other forms. In this study, nine representative reliability indices were selected, and they were analyzed as to how well they reflect changes in hydraulic measures according to abnormal conditions. The following is a description of the concept and specific calculation for each reliability index.

\subsubsection{Hydraulic Reliability Index}

The resilience index (RI) quantifies WDN reliability based on system power such as input, dissipated, and surplus power. In a looped network, the goal is to provide more power (energy per unit time) at each node than is required, in order to have a sufficient surplus to be dissipated internally in case of failures. This surplus can be used to characterize the resilience of the looped network, i.e., its intrinsic capability for overcoming sudden failures [13]. In other words, RI is the ratio of surplus power excluding the minimum required power at the node with the total system power supplied; the detailed calculation method can be expressed as

$$
\mathrm{RI}=\frac{\gamma \sum_{j=1}^{\text {node }} Q_{j}\left(H_{j}-H_{r e q, j}\right)}{\gamma \sum_{s=1}^{n s o u r c e} Q_{s} H_{s}+\gamma \sum_{p=1}^{\text {npump }} Q_{p} H_{p}-\gamma \sum_{j=1}^{\text {nnode }} Q_{j} H_{\text {req }, j}}
$$

where $\gamma$ is the specific weight of water; $Q_{s}$ is the inflow at source $s ; Q_{p}$ is the pumping flow at pump $p$; $H_{s}$ is the total head at source $s ; H_{p}$ is the pumping head at pump $p$; nsource is the number of sources; and npump is the number of pumps.

The modified resilience index (MRI) quantifies the reliability of a WDN based on system power, similarly to RI. In particular, MRI uses only required and surplus power to calculate the index and could be used to compare the uncertainty handling of one network relative to another, which is essential in design and rehabilitation problems [14]. A detailed calculation method for MRI is given as follows:

$$
\mathrm{MRI}=\frac{\gamma \sum_{j=1}^{\text {nnode }} Q_{j}\left(H_{j}-H_{r e q, j}\right)}{\gamma \sum_{j=1}^{n \text { node }} Q_{j} H_{r e q, j}}
$$

The available power index (API) quantifies the WDN reliability using total available power and input power. Here, the available power represents the output power at demand nodes; while the unavailable power includes the power dissipated due to pipe friction losses and various minor losses in the network [15]. The detailed calculation method of API is given as

$$
\mathrm{API}=\frac{\gamma \sum_{j=1}^{\text {nnode }} Q_{j} H_{j}}{\gamma \sum_{s=1}^{n s o u r c e} Q_{s} H_{s}+\gamma \sum_{p=1}^{\text {npump }} Q_{p} H_{p}+\gamma \sum_{t=1}^{\text {ntank }} Q_{t} H_{t}}
$$

where $Q_{t}$ denotes the inflow at tank $t ; H_{t}$ is the total head at tank $t$; and $n$ tank is the number of tanks.

\subsubsection{Topological Reliability Index}

The average degree $(\mathrm{AD})$ is a convenient geometric index for quantifying system reliability based on the number of node and pipe elements. It implies that network reliability is proportional to the diversity of link (i.e., pipe) elements and paths. If a network has too few pipes, there will be many isolated nodes and clusters with a small number of nodes. As more pipes are added to the network, the small clusters are connected to larger clusters [18]. The calculation method of AD can be presented as follows: 


$$
\mathrm{AD}=\frac{2 \times \text { npipe }}{\text { nnode }}
$$

While many topological indices, including AD, quantify connectivity of networks using only the number of nodes and pipes without distinction of their layouts, Latora and Marchiori [17] suggested an index, called network efficiency (NE), that quantifies the average efficiency of paths. The NE can be calculated as an average distance between two generic nodes and is expressed by Equation (11).

$$
N E=\frac{1}{\text { nnode }(\text { nnode }-1)} \sum_{j=1}^{\text {nnode }} \sum_{\substack{j^{*}=1 \\ j \neq j^{*}}}^{\text {nnode }} \frac{1}{d_{j j^{*}}}
$$

where $d_{j j^{*}}$ is the shortest path length from node $j$ to node $j^{*}$.

\subsubsection{Entropic Reliability Index}

Shannon [22] derived the informational entropy function as a statistical measure of the amount of uncertainty that a probability distribution represents. The flow entropy (FE) proposed by Tanyimboh and Templeman [21] is another representative entropic reliability index available for WDNs. Prasad and Tanyimboh [30] suggested that this measure can better represent multi-source networks, and it was observed that, as the FE increases, the network becomes more reliable. The detailed calculation method of FE can be presented by Equations (12)-(15).

$$
\begin{gathered}
\mathrm{FE}=E_{0}+\sum_{j=1}^{\text {nnode }} P_{j} E_{j} \\
P_{j}=\frac{T_{j}}{T} \\
E_{0}=-\sum_{s=1}^{n s o u r c e} \frac{Q_{s}}{T} \ln \left(\frac{Q_{s}}{T}\right) \\
E_{j}=-\frac{Q_{j}}{T_{j}} \ln \left(\frac{Q_{j}}{T_{j}}\right)-\sum_{j i \in N D_{j}} \frac{Q_{j i}}{T_{j}} \ln \left(\frac{Q_{j i}}{T_{j}}\right)
\end{gathered}
$$

where $T_{j}$ is the total flow reaching node $j ; T$ is the sum of the nodal demands; $N D_{j}$ denotes the set of all pipe flows emanating from node $j$; and $Q_{j i}$ represents the flow rate at pipe $i$ from node $j$.

\subsubsection{Mixed Reliability Index}

The pipe hydraulic resilience index (PHRI) focuses on nodal water head and simultaneously considers the hydraulic gradient between upstream and downstream nodes; hence, it can be categorized as a mixed reliability index based on hydraulic and topographical aspects. The detailed calculation method for PHRI can be presented as Equations (16)-(19).

$$
\begin{gathered}
\text { PHRI }=\frac{\sum_{i=1}^{n p i p e}\left(S_{i}\right)}{\sum_{i=1}^{n p i p e}\left(A_{i}+S_{i}\right)} \\
S_{i}=\frac{1}{2}\left(H_{d s, i}-H_{d s, r e q, i}\right) L_{p r o, i} \\
S_{i}+A_{i}=\frac{1}{2}\left(H_{u s, i}-H_{d s, r e q, i}\right) L_{p r o, i}
\end{gathered}
$$




$$
L_{p r o, i}=\sqrt{L_{i}{ }^{2}-\left(Z_{u s, i}-Z_{d s, i}\right)^{2}}
$$

where $H_{d s, i}$ is the total head at the downstream node of pipe $i ; H_{u s, i}$ is the total head at the upstream node of pipe $i ; H_{d s, \text { req }, i}$ is the minimum required head at the downstream node of pipe $i ; L_{i}$ is the length of pipe $i ; Z_{d s, i}$ is the elevation at the downstream node of pipe $i$; and $Z_{u s, i}$ is the elevation at the upstream node of pipe $i$.

The revised resilience index (RRI) is the mixed reliability index based on hydraulic and topographical approaches. Although the calculation method for RRI is identical with MRI, RRI applies the hydraulic gradient representing network topography when calculating the minimum required head at downstream nodes [16]. The calculation method for RRI is as shown in Equation (20).

$$
\mathrm{RRI}=\frac{\gamma \sum_{j=1}^{\text {nnode }} Q_{j}\left(H_{j}-H_{r e q, j}^{*}\right)}{\gamma \sum_{j=1}^{n \text { node }} Q_{j} H_{r e q, j}^{*}}
$$

where $H_{r e q, j}^{*}$ denotes the actual minimum required head at node $j$.

The network resilience index (NRI) is another mixed reliability index incorporating network topology into the formulation of RI. In WDNs, reliable loops can be ensured, if the pipes connected to a node are not widely varying in diameter. NRI incorporates the effects of both surplus power and reliable loops [6]. The detailed calculation method of NRI is as shown in Equations (21) and (22):

$$
\begin{gathered}
\mathrm{NRI}=\frac{\gamma \sum_{j=1}^{\text {nnode }} C_{j} Q_{j}\left(H_{j}-H_{r e q, j}\right)}{\gamma \sum_{s=1}^{\text {nsource }} Q_{s} H_{s}+\gamma \sum_{p=1}^{n \text { nump }} Q_{p} H_{p}-\gamma \sum_{j=1}^{\text {nnode }} Q_{j} H_{\text {req }, j}} \\
C_{j}=\frac{\sum_{i=1}^{\text {npipe }} D_{i}}{\text { npipe }_{j} \times \max \left\{D_{i}\right\}}
\end{gathered}
$$

where $n$ pipe $j$ is the number of pipes connected with node $j$; and $D_{i}$ denotes the diameter of pipe $i$.

\subsection{Multi-Criteria Decision Analysis}

Since each index may fit different scenarios and performance aspects, it can be seen that comprehensive comparative analysis is difficult to achieve. Thus, multi-criteria decision analysis (MCDA) can be applied for drawing quantitative and comprehensive conclusions in complex decisions with multiple criteria. In urban water infrastructure, MCDA techniques such as weighted sum model (WSM), weighted product model (WPM), analytic hierarchy process (AHP), technique for order of preference by similarity to ideal solution (TOPSIS), and 'elimination et choix traduisant la realité' (ELECTRE) are used [31]. Gheisi et al. [32] applied MCDA methods such as WSM, WPM, and TOPSIS to five reliability indices as a way of determining the optimal pipe layout and diameter designs in a hypothetical WDN and claimed that WSM can be easily applied to MCDA of WDN.

\section{Applications}

\subsection{Application Networks}

In this study, to consider various types of network, application networks of various layouts were designed for the same region. Specifically, as shown in Figures 2 and 3, for a region with 25 demand nodes distributed in a grid format, application networks were configured with 17 different layouts, which ranged from the most-looped P-41 layout with all of the 41 pipes arranged to the most-branched P-25 layout with only 25 pipes arranged. All 17 networks supply the 25 demand nodes from a single source with a total head of $45 \mathrm{~m}$. 


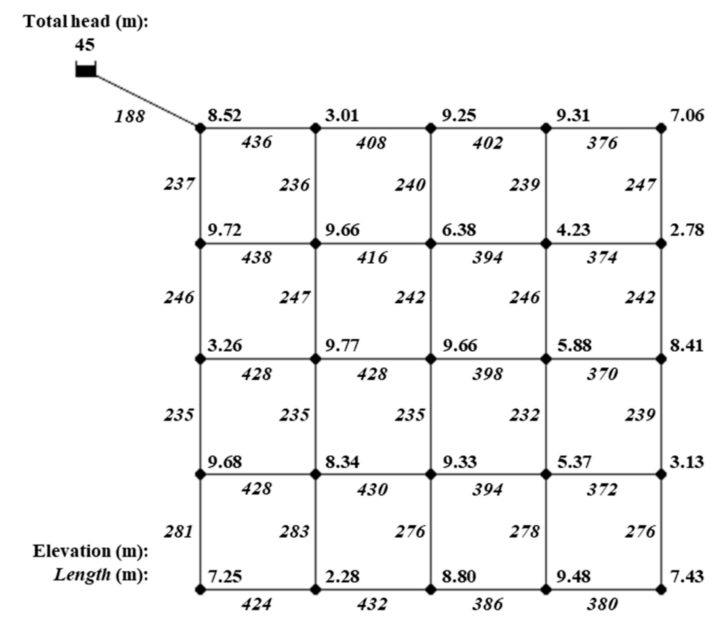

Figure 2. Configuration of application grid network (P-41).

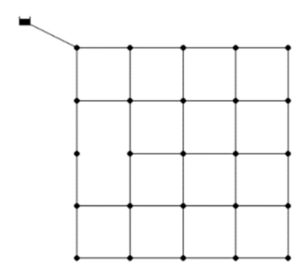

P-40

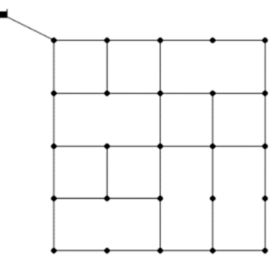

P-36

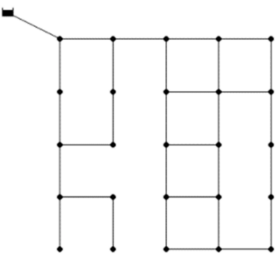

P-32

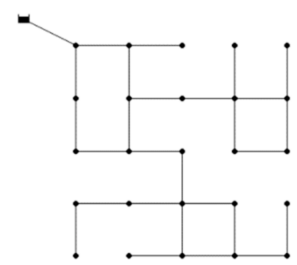

P-28

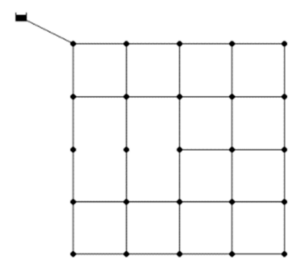

P-39

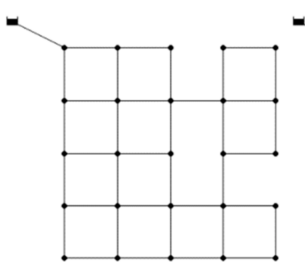

P-38

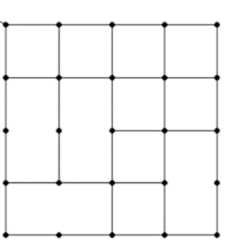

P-37

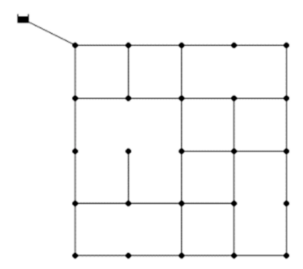

P-35

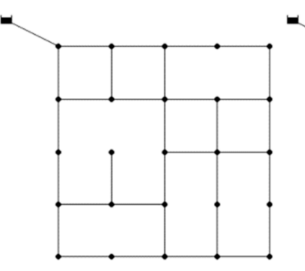

P-34

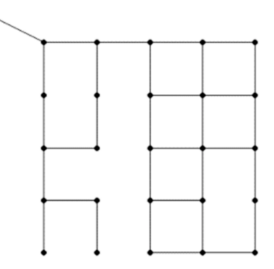

P-33

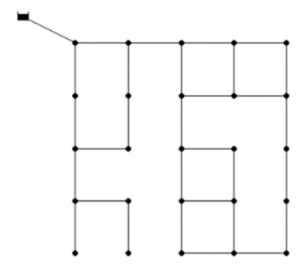

P-31

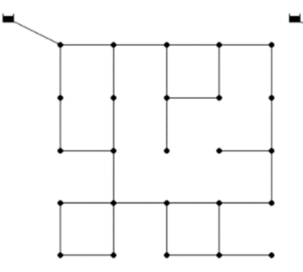

P-30

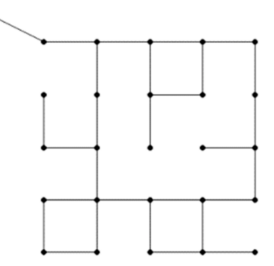

P-29

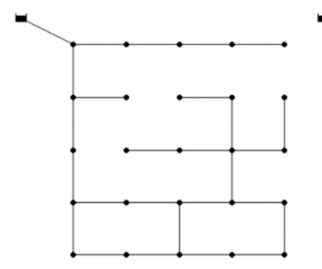

P-27

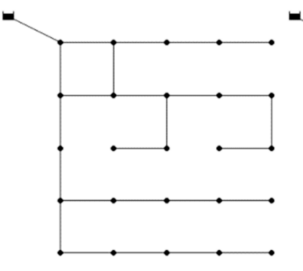

P-26

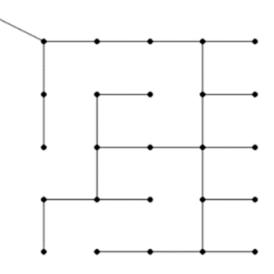

P-25

Figure 3. Optimally designed application network layout. 
Base demand at all nodes was equal at $9.49 \mathrm{~m}^{3} / \mathrm{h}$, and 17 networks were designed by applying an hourly peaking factor (HPF) of 2.25 to produce design flow. Here, a multi-objective genetic algorithm (MOGA) was applied with decision variables of pipe layout and diameters, objective functions of design cost (minimization) and reliability indices (maximization), and a constraint of satisfying the minimum required pressure $(15 \mathrm{~m})$. Finally, 17 optimally designed networks were selected from the P-41 to P-25 layout considering distribution of reliability-index values. Figure 2 shows the node elevation and pipe length of the P-41 network, and the optimal layouts of the P-40-P-25 networks can be comprehensively viewed in Figure 3 .

\subsection{Application Scenarios}

In this study, four scenarios, including a normal condition, were constructed to simulate performance change under abnormal conditions. The simulation of each scenario was performed using the pressure driven analysis (PDA) module provided by EPANET 3 [33], a WDN analysis software. The specific water supply conditions according to the four scenarios applied in this study are described in the following subsections.

\subsubsection{Base Scenario}

In the base scenario corresponding to a normal operating condition, a daily peaking factor (DPF) of 1.80 was applied to the base demand to simulate daily water usage. The hydraulic measures under the base scenario serve as the reference values of performance changes according to the subsequent abnormal scenarios and are used for calculation of the nine reliability indices as summarized in Table 1. Note that the higher reliability index value indicates a higher system reliability.

Table 1. Reliability index values of 17 application networks in the base scenario.

\begin{tabular}{cccccccccc}
\hline \multirow{2}{*}{ Network Layout } & \multicolumn{8}{c}{ Reliability Index } \\
\cline { 2 - 9 } & RI & MRI & API & AD & NE & FE & PHRI & RRI & NRI \\
\hline P-25 & 0.31 & 0.32 & 0.65 & 2.00 & 0.59 & 1.18 & 0.77 & 0.06 & 0.26 \\
P-26 & 0.40 & 0.41 & 0.70 & 2.08 & 0.54 & 1.55 & 0.84 & 0.07 & 0.36 \\
P-27 & 0.40 & 0.42 & 0.70 & 2.16 & 0.60 & 2.26 & 0.81 & 0.04 & 0.35 \\
P-28 & 0.41 & 0.43 & 0.70 & 2.24 & 0.60 & 2.14 & 0.82 & 0.15 & 0.35 \\
P-29 & 0.55 & 0.57 & 0.77 & 2.32 & 0.64 & 3.74 & 0.85 & 0.16 & 0.46 \\
P-30 & 0.62 & 0.64 & 0.81 & 2.40 & 0.70 & 3.04 & 0.88 & 0.17 & 0.52 \\
P-31 & 0.50 & 0.52 & 0.75 & 2.48 & 0.71 & 4.16 & 0.90 & 0.19 & 0.41 \\
P-32 & 0.51 & 0.53 & 0.75 & 2.56 & 0.77 & 4.99 & 0.90 & 0.20 & 0.41 \\
P-33 & 0.56 & 0.58 & 0.77 & 2.64 & 0.78 & 5.33 & 0.92 & 0.27 & 0.44 \\
P-34 & 0.81 & 0.84 & 0.90 & 2.72 & 0.80 & 5.93 & 0.96 & 0.59 & 0.66 \\
P-35 & 0.82 & 0.85 & 0.91 & 2.80 & 0.91 & 5.92 & 0.96 & 0.58 & 0.64 \\
P-36 & 0.84 & 0.87 & 0.92 & 2.88 & 0.86 & 6.21 & 0.97 & 0.61 & 0.66 \\
P-37 & 0.80 & 0.82 & 0.90 & 2.96 & 0.94 & 6.81 & 0.96 & 0.53 & 0.61 \\
P-38 & 0.73 & 0.75 & 0.86 & 3.04 & 0.89 & 8.82 & 0.94 & 0.41 & 0.57 \\
P-39 & 0.88 & 0.91 & 0.94 & 3.12 & 0.97 & 8.93 & 0.98 & 0.60 & 0.69 \\
P-40 & 0.89 & 0.92 & 0.94 & 3.20 & 0.98 & 8.98 & 0.98 & 0.67 & 0.69 \\
P-41 & 0.89 & 0.92 & 0.94 & 3.28 & 0.99 & 9.22 & 0.98 & 0.69 & 0.74 \\
\hline
\end{tabular}

RI: resilience index, MRI: modified resilience index, API: available power index, AD: average degree, NE: network efficiency, FE: flow entropy, PHRI: pipe hydraulic resilience index, RRI: revised resilience index, NRI: network resilience index.

\subsubsection{Scenario 1: Single-Pipe Failure}

In Scenario 1, based on the base scenario flow condition, a single-pipe failure, in which each pipe is sequentially closed one by one, is constructed. Here, since all the pipes are closed once each, the probability of failure can be assumed to be the same. Therefore, changes in the performance of 
the entire system were calculated by averaging the measured changes of individual nodes over all failure cases.

\subsubsection{Scenario 2: Water Consumption Increase}

In Scenario 2, a scenario of increasing water consumption according to climate change and population growth was assumed. A study by Pachauri and Meyer [34] predicted that the global average temperature would increase by about $2{ }^{\circ} \mathrm{C}$ by 2050 according to the RCP (Representative Concentration Pathway) 8.5 climate change scenario of the Intergovernmental Panel on Climate Change (IPCC). Kenney et al. [35] found that daily water consumption increased by $2 \%$ for average temperature increases of $0.56{ }^{\circ} \mathrm{C}$. Furthermore, Hoornweg and Pope [36] predicted that the population of major metropolitan cities in the world will increase by about $43.1 \%$ from 2025 to 2050, according to the shared socioeconomic pathways (SSP) scenario. Therefore, in this scenario, it is assumed that the base demand will increase by $41.5 \%$, from 9.49 to $13.43 \mathrm{~m}^{3} / \mathrm{h}$, according to the climate change and population growth prediction. Here, a DPF of 1.80 was also applied to establish the daily water usage condition.

\subsubsection{Scenario 3: Fire Flow}

In Scenario 3, adding to the base scenario flow condition, fire flow demands are sequentially generated at each node. It is assumed that fire occurs once for each node, and the probability of fire outbreak is the same in all cases; therefore, the performance change according to Scenario 3 can be identified through the average of the measured changes according to the simulation of individual fire flows. The Ontario Ministry of the Environment and Climate Change (OMOECC) [37] estimated fire flow demand according to the supply population of a WDN as shown in Table 2. Since the equivalent population of each node according to the base demand of the application network is approximately 1000 , the fire flow demand at each node was applied as $230.4 \mathrm{~m}^{3} / \mathrm{h}$. Table 3 presents a brief summary of each design condition, the base scenario and the three abnormal scenarios introduced above.

Table 2. Estimation of fire flow requirements (OMOECC [37]).

\begin{tabular}{ccc}
\hline Equivalent Population & Nodal Base Demand $(\mathbf{m} 3 / \mathbf{h})$ & Suggested Fire Flow $(\mathbf{m} 3 / \mathbf{h})$ \\
\hline $500-1000$ & 4.6 & 136.8 \\
1000 & 9.2 & 230.4 \\
1500 & 13.8 & 284.4 \\
2000 & 18.3 & 342.0 \\
3000 & 27.5 & 396.0 \\
4000 & 36.7 & 450.0 \\
5000 & 45.8 & 518.4 \\
6000 & 55.0 & 572.4 \\
\hline
\end{tabular}

Table 3. Application scenarios comparison.

\begin{tabular}{ccccc}
\hline Scenario & $\begin{array}{c}\text { Base Demand per } \\
\text { Node }\left(\mathbf{m}^{\mathbf{3}} \mathbf{h}\right)\end{array}$ & $\begin{array}{c}\text { Peaking } \\
\text { Factor }\end{array}$ & $\begin{array}{c}\text { Peak Demand per } \\
\text { Node }\left(\mathbf{m}^{\mathbf{3}} \mathbf{h}\right)\end{array}$ & Description \\
\hline Design & 9.49 & 2.25 & 21.35 & Network design condition \\
Base & 9.49 & 1.80 & 17.08 & Base condition for normal operation \\
Scenario 1 & 9.49 & 1.80 & 17.08 & Single-pipe failure \\
Scenario 2 & 13.43 & 1.80 & 24.17 & Water consumption increase \\
Scenario 3 & 9.49 & 1.80 & 17.08 & Fire flow at single node \\
\hline
\end{tabular}

\section{Results}

\subsection{Correlation between Reliability Indices and Hydraulic Measures}

For 17 application networks, a total of 54 correlations were analyzed through the results of nine reliability indices calculated in the base scenario and the results of six hydraulic measures collected 
in Scenarios 1-3. As all reliability indices and hydraulic measures indicate that the higher the value, the more superior the reliability and performance of the network, it can be concluded that the larger the derived correlation coefficient, the better the index is at reflecting system performance.

\subsubsection{Results for Scenario 1: Single-Pipe Failure}

Figure 4 and Table 4 show the correlation between reliability indices and hydraulic measures according to the application of Scenario 1. In Figure 4, the points in the scatter plot represent the reliability index values and hydraulic measures derived from each of the 17 networks ( $x$-axis-Reliability index; $y$-axis-Hydraulic measure), and Table 4 summarizes the correlation coefficients calculated from each scatter plot. The plots and table values indicated in yellow are index-measure combinations with relatively high correlation coefficients of 0.95 or higher.
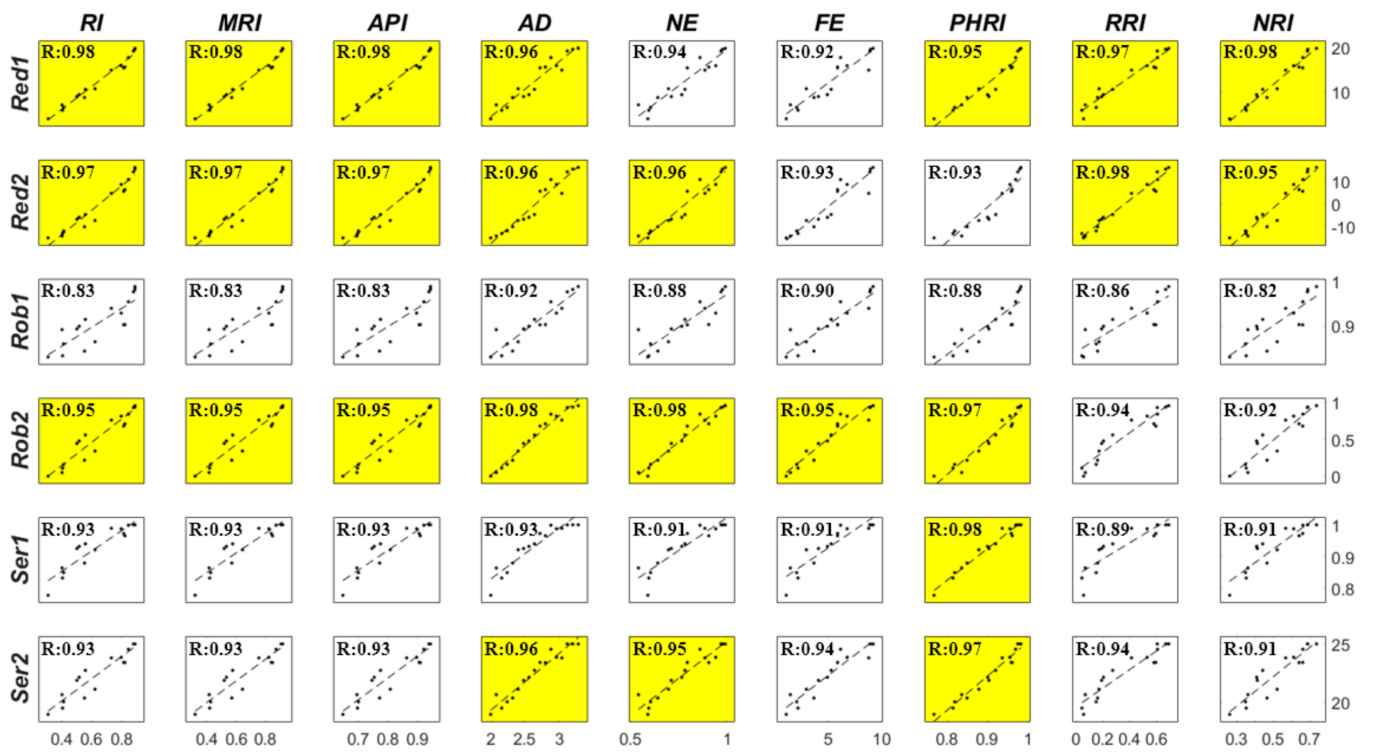

Figure 4. Scatter plots of Scenario 1 ( $x$-axis-Reliability index; $y$-axis-Hydraulic measures). The plots highlighted in yellow show high correlation coefficient of 0.95 or higher.

Table 4. Correlation coefficients for Scenario 1.

\begin{tabular}{ccccccccccc}
\hline \multirow{2}{*}{ Hydraulic Measure } & \multicolumn{10}{c}{ Reliability Index } \\
\cline { 3 - 11 } & & RI & MRI & API & AD & NE & FE & PHRI & RRI & NRI \\
\hline \multirow{2}{*}{ Redundancy } & Red1 & $\mathbf{0 . 9 8}$ & $\mathbf{0 . 9 8}$ & $\mathbf{0 . 9 8}$ & $\mathbf{0 . 9 6}$ & 0.94 & 0.92 & $\mathbf{0 . 9 5}$ & $\mathbf{0 . 9 7}$ & $\mathbf{0 . 9 8}$ \\
& Red2 & $\mathbf{0 . 9 7}$ & $\mathbf{0 . 9 7}$ & $\mathbf{0 . 9 7}$ & $\mathbf{0 . 9 6}$ & $\mathbf{0 . 9 6}$ & 0.93 & 0.93 & $\mathbf{0 . 9 8}$ & $\mathbf{0 . 9 5}$ \\
\multirow{3}{*}{ Robustness } & Rob1 & 0.83 & 0.83 & 0.83 & 0.92 & 0.88 & 0.90 & 0.88 & 0.86 & 0.82 \\
& Rob2 & $\mathbf{0 . 9 5}$ & $\mathbf{0 . 9 5}$ & $\mathbf{0 . 9 5}$ & $\mathbf{0 . 9 8}$ & $\mathbf{0 . 9 8}$ & $\mathbf{0 . 9 5}$ & $\mathbf{0 . 9 7}$ & 0.94 & 0.92 \\
\multirow{3}{*}{ Serviceability } & Ser1 & 0.93 & 0.93 & 0.93 & 0.93 & 0.91 & 0.91 & $\mathbf{0 . 9 8}$ & 0.89 & 0.91 \\
& Ser2 & 0.93 & 0.93 & 0.93 & $\mathbf{0 . 9 6}$ & $\mathbf{0 . 9 5}$ & 0.94 & $\mathbf{0 . 9 7}$ & 0.94 & 0.91 \\
\hline
\end{tabular}

Note: The values highlighted in yellow indicate high correlation coefficient of 0.95 or higher.

In Scenario 1, a broken pipe causes increment of flow and head loss to substitute paths. The hydraulic indices (RI, MRI, and API) are proportional to excessive nodal pressure in normal water demand conditions. When the pipe is broken, the network which has bigger excessive pressure remains more backup pressure after the event, thus the redundancy measures (Red1 and Red2) have relatively high correlation with the hydraulic indices ( $R$ values are 0.98 and 0.97 , respectively).

The topological indices (AD and NE) quantify the diversity of water paths. For the pipe failure scenario, therefore, these indices show relatively high correlation with all measures indicating good 
representation of system performance under the specific condition ( $R$ values are higher than 0.95 for Red2, Rob2, and Ser2).

The entropic index (FE) quantifies the diversity and relative uniformity of flow distribution. When a pipe is isolated, the higher flow diversity leads to better adaptation against rapid increase of water flow. However, increased flow occurs in limited local paths, thus the overall correlation with the measures is relatively low compared to other indices.

Among the mixed indices (PHRI, RRI, and NRI), PHRI shows good correlation, especially with the serviceability measures ( $R$ values are 0.98 for Ser1 and 0.97 for Ser2). For a pipe isolation condition, a network with a lower hydraulic gradient gets better service. RRI and NRI are calculated in a similar way to RI, thus, they also show high correlation with the redundancy measures, but have a relatively low correlation with other measures.

\subsubsection{Results for Scenario 2: Water Consumption Increase}

Figure 5 and Table 5 show the correlation between reliability indices and hydraulic measures according to the application of Scenario 2. In Scenario 2, system-wide increased water consumption causes an increment of flow and head loss in all pipes; that is, a system-wide stress is produced under this scenario. This mainly affects the branch-type networks (P-25-P-33) and significantly reduces the measures of Red2 and Rob2 that are related to the minimum nodal pressure as seen in Figure 5 (see scatter plots in second and fourth rows).
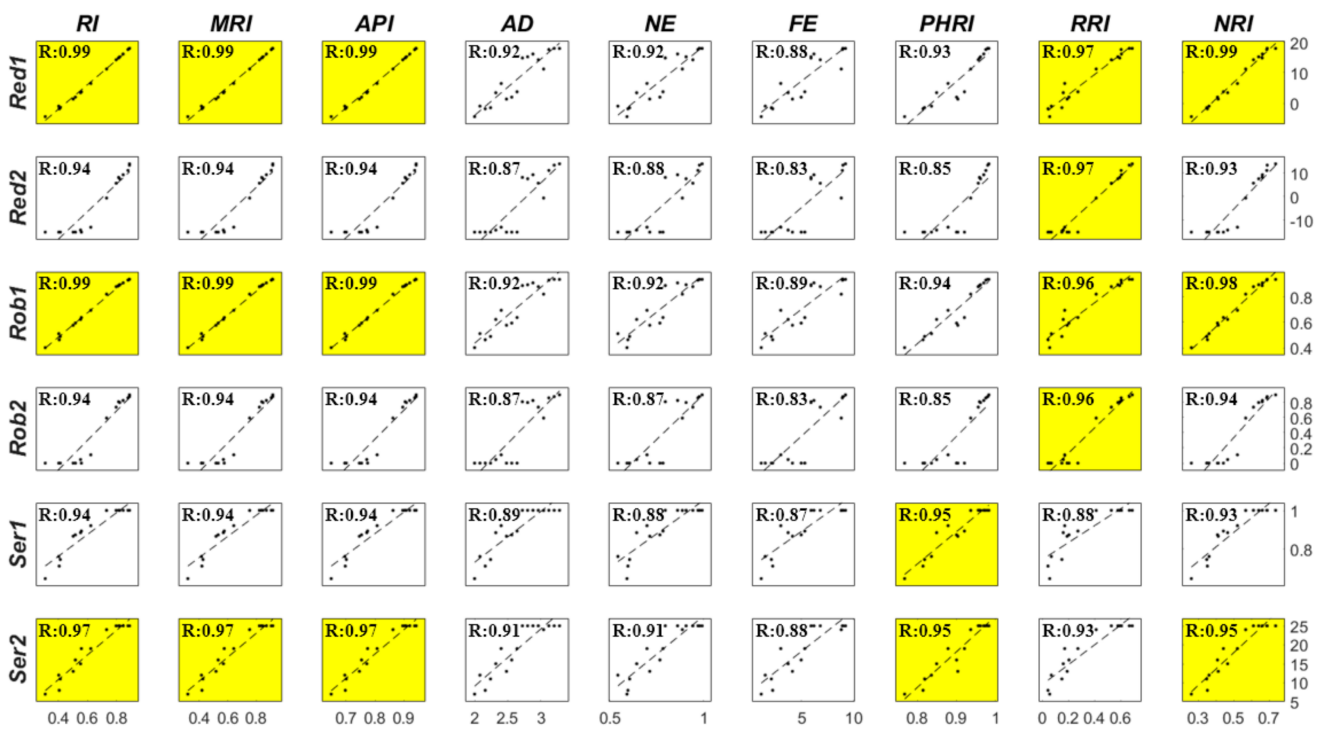

Figure 5. Scatter plots of Scenario 2 ( $x$-axis—Reliability index; $y$-axis-Hydraulic measures). The plots highlighted in yellow show high correlation coefficient of 0.95 or higher.

Table 5. Correlation coefficients for Scenario 2.

\begin{tabular}{ccccccccccc}
\hline \multirow{2}{*}{ Hydraulic Measure } & \multicolumn{10}{c}{ Reliability Index } \\
\cline { 3 - 10 } & & RI & MRI & API & AD & NE & FE & PHRI & RRI & NRI \\
\hline \multirow{2}{*}{ Redundancy } & Red1 & $\mathbf{0 . 9 9}$ & $\mathbf{0 . 9 9}$ & $\mathbf{0 . 9 9}$ & 0.92 & 0.92 & 0.88 & 0.93 & $\mathbf{0 . 9 7}$ & $\mathbf{0 . 9 9}$ \\
& Red2 & 0.94 & 0.94 & 0.94 & 0.87 & 0.88 & 0.83 & 0.85 & $\mathbf{0 . 9 7}$ & 0.93 \\
\multirow{3}{*}{ Robustness } & Rob1 & $\mathbf{0 . 9 9}$ & $\mathbf{0 . 9 9}$ & $\mathbf{0 . 9 9}$ & 0.92 & 0.92 & 0.89 & 0.94 & $\mathbf{0 . 9 6}$ & $\mathbf{0 . 9 8}$ \\
& Rob2 & 0.94 & 0.94 & 0.94 & 0.87 & 0.87 & 0.83 & 0.85 & $\mathbf{0 . 9 6}$ & 0.94 \\
\multirow{2}{*}{ Serviceability } & Ser1 & 0.94 & 0.94 & 0.94 & 0.89 & 0.88 & 0.87 & $\mathbf{0 . 9 5}$ & 0.88 & 0.93 \\
& Ser2 & $\mathbf{0 . 9 7}$ & $\mathbf{0 . 9 7}$ & $\mathbf{0 . 9 7}$ & 0.91 & 0.91 & 0.88 & $\mathbf{0 . 9 5}$ & 0.93 & $\mathbf{0 . 9 5}$ \\
\hline
\end{tabular}

Note: The values highlighted in yellow indicate high correlation coefficient of 0.95 or higher. 
The hydraulic indices (RI, MRI, and API) show great correlations with the measures overall (in range of 0.94-0.99) under Scenario 2. The higher value of the hydraulic indices implies that the networks have excessive pressure, thus providing redundancy, guaranteeing maintenance of pressure, and allowing better serviceability.

In contrast, the topological indices (AD and NE) and entropic index (FE) show relatively low correlation with the performance measures under Scenario 2 in which overall flows increase without changes in network connectivity/layout and flow paths.

For the mixed indices, PHRI quantifies the serviceability well, while RRI represents the redundancy and robustness measures well. It is noteworthy that RRI has a strong correlation with Red2 and Rob2 ( $R$ values are 0.97 and 0.96, respectively), which cannot be seen in other indices under Scenario 2.

\subsubsection{Results for Scenario 3: Fire Flow}

Figure 6 and Table 6 show the correlation between reliability indices and hydraulic measures according to the application of Scenario 3. In Scenario 3, node-assigned fire flow causes increment of flow and head loss along a specific flow path from the source to the node at which the fire occurred. As seen in Figure 6 and Table 6, overall, the correlation coefficients in Scenario 3 are less than those of the other two abnormal scenarios.
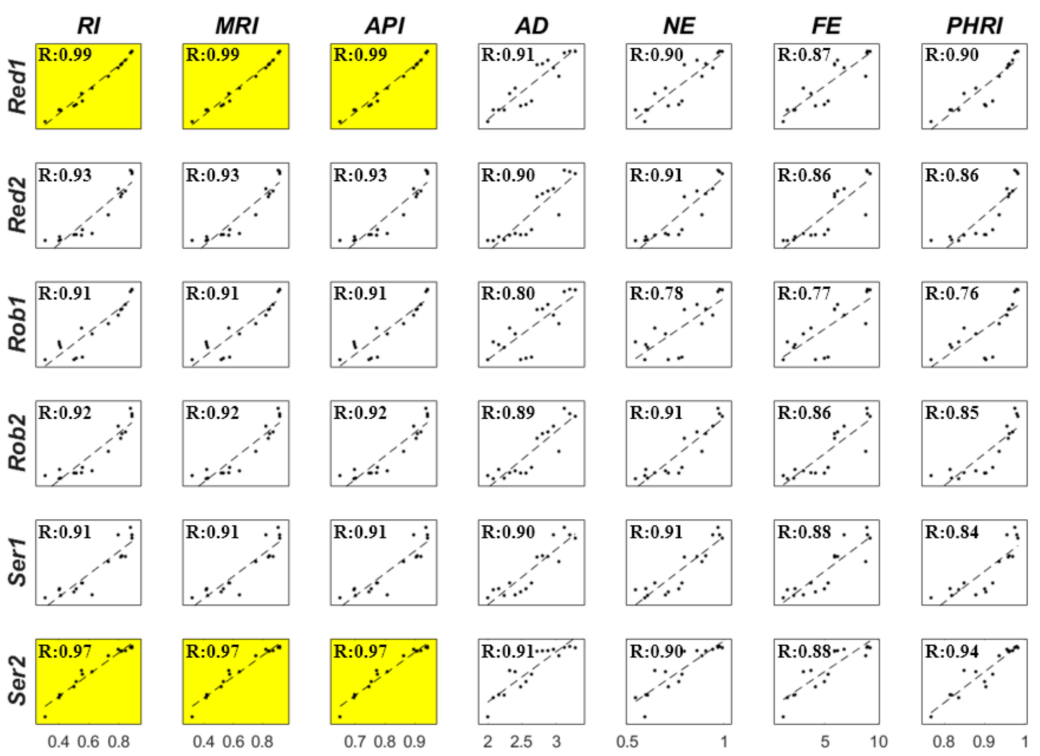
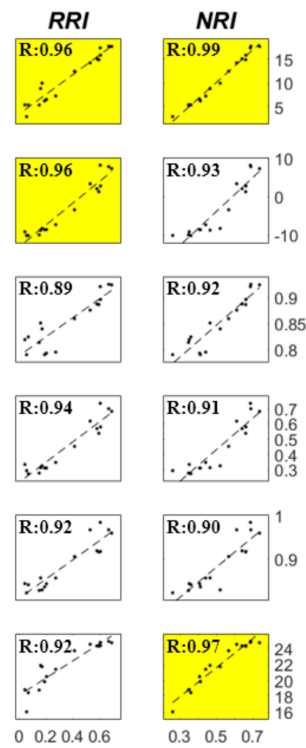

Figure 6. Scatter plots of Scenario 3 ( $x$-axis-Reliability index; $y$-axis-Hydraulic measure). The plots highlighted in yellow show high correlation coefficient of 0.95 or higher.

Table 6. Correlation coefficients for Scenario 3.

\begin{tabular}{ccccccccccc}
\hline \multirow{2}{*}{ Hydraulic Measure } & \multicolumn{10}{c}{ Reliability Index } \\
\cline { 3 - 10 } & & RI & MRI & API & AD & NE & FE & PHRI & RRI & NRI \\
\hline \multirow{2}{*}{ Redundancy } & Red1 & $\mathbf{0 . 9 9}$ & $\mathbf{0 . 9 9}$ & $\mathbf{0 . 9 9}$ & 0.91 & 0.90 & 0.87 & 0.90 & $\mathbf{0 . 9 6}$ & $\mathbf{0 . 9 9}$ \\
& Red2 & 0.93 & 0.93 & 0.93 & 0.90 & 0.91 & 0.86 & 0.86 & $\mathbf{0 . 9 6}$ & 0.93 \\
\multirow{3}{*}{ Robustness } & Rob1 & 0.91 & 0.91 & 0.91 & 0.80 & 0.78 & 0.77 & 0.76 & 0.89 & 0.92 \\
& Rob2 & 0.92 & 0.92 & 0.92 & 0.89 & 0.91 & 0.86 & 0.85 & 0.94 & 0.91 \\
\multirow{2}{*}{ Serviceability } & Ser1 & 0.91 & 0.91 & 0.91 & 0.90 & 0.91 & 0.88 & 0.84 & 0.92 & 0.90 \\
& Ser2 & $\mathbf{0 . 9 7}$ & $\mathbf{0 . 9 7}$ & $\mathbf{0 . 9 7}$ & 0.91 & 0.90 & 0.88 & 0.94 & 0.92 & $\mathbf{0 . 9 7}$ \\
\hline
\end{tabular}

Note: The values highlighted in yellow indicate high correlation coefficient of 0.95 or higher. 
Overall, the hydraulic indices (RI, MRI, and API) and mixed hydraulic index (NRI) show higher correlations ( $R$ values are in the range of 0.90-0.99) compared to other indices (in the range of 0.76-0.91). It is interesting to observe that RRI shows strong correlation with Red2 and Rob2 under Scenarios $1-3$ ( $R$ values are always ranged in $0.94-0.98$ ). It should be noted that Red2 and Rob2 measure the minimum pressure at critical nodes and RRI represents the resilience of the network while maintaining the minimum required head.

The topological indices (AD and NE), entropic index (FE), and a hydraulic-gradient based mixed index (PHRI) show relatively low correlation with the measures under the fire-flow scenario; none of them yield a correlation coefficient of greater than 0.95 .

\subsection{Multi Criteria Decision Analysis (MCDA)}

In this study, MCDA was performed using WSM by assigning equal weights to all six performance measures. Here, two different MCDAs are conducted: a scenario-based MCDA and a performance-based MCDA. The scenario-based MCDA aims to find the optimal indices representing the individual abnormal conditions; while the performance-based MCDA intends to find the best fit indices for individual performance such as redundancy, robustness, and serviceability. Finally, a comprehensive evaluation of individual indices is achieved by combining both MCDA results.

\subsubsection{Scenario-Based MCDA}

Table 7 shows the MCDA results of nine indices according to different scenarios. In Scenario 1, $\mathrm{AD}$ and PHRI showed high correlation coefficients from MCDA, which indicates that these indices appropriately quantify the system performance under the single pipe failure condition. In a similar way, under Scenario 2 (system-wide water consumption increase), hydraulic indices (RI, MRI, and API) show great performance; while for Scenario 3 (single-node fire flow), the hydraulic indices still show better performance than other indices but, overall, correlation coefficients were lower than those of the other scenarios.

Table 7. Correlation coefficients obtained by scenario-based MCDA.

\begin{tabular}{cccccccccc}
\hline \multirow{2}{*}{ Abnormal Scenario } & \multicolumn{10}{c}{ Reliability Index } \\
\cline { 2 - 10 } & RI & MRI & API & AD & NE & FE & PHRI & RRI & NRI \\
\hline Scenario 1 & 0.93 & 0.93 & 0.93 & $\mathbf{0 . 9 5}$ & 0.94 & 0.93 & $\mathbf{0 . 9 5}$ & 0.93 & 0.92 \\
Scenario 2 & $\mathbf{0 . 9 6}$ & $\mathbf{0 . 9 6}$ & $\mathbf{0 . 9 6}$ & 0.90 & 0.90 & 0.86 & 0.91 & $\mathbf{0 . 9 5}$ & $\mathbf{0 . 9 5}$ \\
Scenario 3 & 0.94 & 0.94 & 0.94 & 0.89 & 0.89 & 0.85 & 0.86 & 0.93 & 0.94 \\
\hline Average & 0.94 & 0.94 & 0.94 & 0.91 & 0.91 & 0.88 & 0.91 & 0.94 & 0.94 \\
\hline
\end{tabular}

Note: The values highlighted in yellow indicate high correlation coefficient of 0.95 or higher.

\subsubsection{Performance-Based MCDA}

Table 8 shows the MCDA results of nine indices according to the system performance. For system redundancy quantification, the hydraulic indices (RI, MRI, and API) and two mixed indices (RRI, and NRI) showed great performance in all scenarios. Regarding the robustness context, the overall correlation coefficients are relatively low, which indicates that the applied indices are inadequate to represent the system robustness under the applied scenarios. For measuring system serviceability, the hydraulic indices and PHRI, showed relatively good results.

Finally, the combined MCDA results can be obtained via the averages of Tables 7 and 8 (assuming equal weights for WSM). As seen, among the applied nine indices, the hydraulic indices (RI, MRI, and API) and the hydraulic-based mixed indices (RRI, and NRI) showed high correlation coefficients (i.e., 0.94) and are considered as the optimal indices to quantify the system performance under the applied abnormal scenarios and hydraulic measures. 
Table 8. Correlation coefficients obtained by performance-based MCDA.

\begin{tabular}{cccccccccc}
\hline \multirow{2}{*}{ System Performance } & \multicolumn{10}{c}{ Reliability Index } \\
\cline { 2 - 10 } & RI & MRI & API & AD & NE & FE & PHRI & RRI & NRI \\
\hline Redundancy & $\mathbf{0 . 9 7}$ & $\mathbf{0 . 9 7}$ & $\mathbf{0 . 9 7}$ & 0.92 & 0.92 & 0.88 & 0.90 & $\mathbf{0 . 9 7}$ & $\mathbf{0 . 9 6}$ \\
Robustness & 0.92 & 0.92 & 0.92 & 0.90 & 0.89 & 0.87 & 0.88 & 0.93 & 0.92 \\
Serviceability & 0.94 & 0.94 & 0.94 & 0.92 & 0.91 & 0.89 & 0.94 & 0.91 & 0.93 \\
\hline Average & 0.94 & 0.94 & 0.94 & 0.91 & 0.91 & 0.88 & 0.91 & 0.94 & 0.94 \\
\hline
\end{tabular}

Note: The values highlighted in yellow indicate high correlation coefficient of 0.95 or higher.

\section{Conclusions}

In this study, correlations between hydraulic measures and reliability indices were analyzed to evaluate the ability of reliability indices to reflect system performance. To that purpose, six (hydraulic) measures of redundancy, robustness, and serviceability and nine reliability indices based on hydraulic, topological, entropic, and mixed approaches were selected. Correlation analyses were performed using the 17 optimally designed hypothetic WDNs under three abnormal operation scenarios. To obtain a comprehensive evaluation of individual indices, an MCDA based on WSM was performed as a post-analysis. Based on the results obtained in the study, the following conclusions can be drawn:

1. In the single-pipe-failure scenario, the topological indices (AD and NE) and mixed index (PHRI) were found to be the best at the quantification of network performance.

2. In the demand increase scenario, the hydraulic or mixed hydraulic indices (RI, MRI, API, RRI, and NRI) were found to be the best at representation of network performance.

3. In the fire flow scenario, the hydraulic indices (RI, MRI, and API) were found to be best at representation of network performance in terms of redundancy and serviceability. It was found that NRI represents very close correlation trends with the hydraulic indices in all scenarios.

4. For redundancy quantification, the hydraulic or mixed hydraulic indices (RI, MRI, API, RRI, and NRI) showed the best performance.

5. For robustness quantification, all indices showed relatively low correlation with the measures, indicating that the applied indices do not sufficiently reflect system robustness.

6. For serviceability quantification, the hydraulic indices (RI, MRI, and API) and PHRI were found to best reflect network performance.

7. From the combined MCDA, the hydraulic or mixed hydraulic indices (RI, MRI, API, RRI, and NRI) were found to be the optimal indices to quantify the system performance under the applied abnormal scenarios.

Prior to using any reliability index for the design and operation of a WDN, it is essential to select an adequate reliability index suitable for the goals of the designer/operator, and the results of this study can contribute to that purpose. In addition, the proposed comparative analyses are expected to be useful in research on index development that can better reflect various network performances. Future study should consider the needs of water managers for scenario development reflecting real operational conditions and the selection of reliability indices and hydraulic measures. Further comparative analyses, including various reliability indices and hydraulic measures for a number of WDNs with diverse sizes and layouts, are a fruitful area to pursue as a future study. Through the proposed comparative analysis, it is anticipated that the best fit reliability indices can be suggested and developed for quantification of WDN performance.

Author Contributions: Conceptualization, G.J. and D.K.; methodology, G.J. and D.K.; software, G.J.; validation, D.K.; formal analysis, G.J. and D.K.; writing—original draft preparation, G.J.; writing—review and editing, D.K.; supervision, D.K.; funding acquisition, D.K. All authors have read and agreed to the published version of the manuscript. 
Funding: This work was supported by the National Research Foundation of Korea (NRF) grant funded by the Korea government (MSIT-Ministry of Science and ICT) (No. NRF-2020R1A2C2009517).

Conflicts of Interest: The authors declare no conflict of interest.

\section{References}

1. Wildavsky, A. Searching for Safety; Transaction Books: New Brunswick, NJ, Canada, 1988.

2. Comfort, L. Shared Risk: Complex Systems in Seismic Response; Pergamon: New York, NY, USA, 1999.

3. Maier, H.R.; Lence, B.J.; Tolson, B.A.; Foschi, R.O. First-order reliability method for estimating reliability, vulnerability, and resilience. Water Resour. Res. 2001, 37, 779-790. [CrossRef]

4. Bruneau, M.; Chang, S.E.; Eguchi, R.T.; Lee, G.C.; O’Rourke, T.D.; Reinhorn, A.M.; Von Winterfeldt, D. A framework to quantitatively assess and enhance the seismic resilience of communities. Earthq. Spectra 2003, 19, 733-752. [CrossRef]

5. Xu, C.; Goulter, C. Reliability-based optimal design of water distribution networks. J. Water Resour. Plan. Manag. 1999, 125, 352-362. [CrossRef]

6. Prasad, T.D.; Park, N.S. Multiobjective genetic algorithms for design of water distribution networks. J. Water Resour. Plan. Manag. 2003, 1, 73-82. [CrossRef]

7. Creaco, E.; Franchini, M.; Todini, E. The combined use of resilience and loop diameter uniformity as a good indirect measure of network reliability. Urban Water J. 2016, 13, 167-181. [CrossRef]

8. Marlim, M.S.; Jeong, G.; Kang, D. Identification of Critical Pipes Using a Criticality Index in Water Distribution Networks. Appl. Sci. 2019, 9, 4052. [CrossRef]

9. Markov, I.; Grigoriu, M.; O'Rourke, T.D. An Evaluation of Seismic Serviceability of Water Supply Networks with Application to the San Francisco Auxiliary Water Supply System; NCEER: Buffalo, NY, USA, 1994.

10. Wagner, J.M.; Shamir, U.; Marks, D.H. Water distribution reliability: Simulation methods. J. Water Resour. Plan. Manag. 1998, 114, 276-294. [CrossRef]

11. Mays, L.W. Reliability Analysis of Water Distribution Systems; ASCE: Reston, VA, USA, 1989.

12. Ostfeld, A. Reliability analysis of water distribution systems. J. Hydroinform. 2004, 6, 281-294. [CrossRef]

13. Todini, E. Looped water distribution networks design using a resilience index based heuristic approach. Urban Water 2000, 2, 115-122. [CrossRef]

14. Jayaram, N.; Srinivasan, K. Performance-based optimal design and rehabilitation of water distribution networks using life cycle costing. Water Resour. Res. 2008, 44, W01417. [CrossRef]

15. Liu, H.; Savić, D.A.; Kapelan, Z.; Creaco, E.; Yuan, Y. Reliability surrogate measures for water distribution system design: Comparative analysis. J. Water Resour. Plan. Manag. 2017, 143, 04016072. [CrossRef]

16. Jeong, G.; Wicaksono, A.; Kang, D. Revisiting the resilience index for water distribution networks. J. Water Resour. Plan. Manag. 2017, 143, 04017035. [CrossRef]

17. Latora, V.; Marchiori, M. Efficient behavior of small-world networks. Phys. Rev. Lett. 2016, 87, 198701. [CrossRef] [PubMed]

18. Costa, L.D.F.; Rodrigues, F.A.; Travieso, G.; Villas Boas, P.R. Characterization of complex networks: A survey of measurements. Adv. Phys. 2007, 56, 167-242. [CrossRef]

19. Jamakovic, A.; Uhlig, S. On the relationships between topological measures in real-world networks. Netw. Heterog. Media 2008, 3, 345-359. [CrossRef]

20. Awumah, K.; Goulter, I.; Bhatt, S.K. Assessment of reliability in water distribution networks using entropy based measures. Stoch. Hydrol. Hydraul. 1990, 4, 309-320. [CrossRef]

21. Tanyimboh, T.T.; Templeman, A.B. Calculating maximum entropy flows in networks. J. Oper. Res. Soc. 1993, 44, 383-396. [CrossRef]

22. Shannon, C.E. A mathematical theory of communication. Bell Syst. Tech. J. 1948, 27, 379-423, $623-656$.

23. Raad, D.N.; Sinske, A.N.; Van Vuuren, J.H. Comparison of four reliability surrogate measures for water distribution systems design. Water Resour. Res. 2010, 46, W05524. [CrossRef]

24. Jeong, G.; Kang, D. Hydraulic Uniformity Index for Water Distribution Networks. J. Water Resour. Plan. Manag. 2020, 146, 04019078. [CrossRef]

25. Paez, D.; Filion, Y.; Suribabu, C.R. Correlation analysis of reliability surrogate measures in real size water distribution networks. In Proceedings of the WDSA/CCWI Joint Conference Proceedings, Kingston, ON, Canada, 23-25 July 2018; Volume 1. 
26. Tanyimboh, T.T.; Siew, C.; Saleh, S.; Czajkowska, A. Comparison of surrogate measures for the reliability and redundancy of water distribution systems. Water Resour. Manag. 2016, 30, 3535-3552. [CrossRef]

27. Sirsant, S.; Reddy, M.J. Assessing the Performance of Surrogate Measures for Water Distribution Network Reliability. J. Water Resour. Plan. Manag. 2020, 146, 04020048. [CrossRef]

28. Yazdani, A.; Otoo, R.A.; Jeffrey, P. Resilience enhancing expansion strategies for water distribution systems: A network theory approach. Environ. Model. Softw. 2011, 26, 1574-1582. [CrossRef]

29. Cimellaro, G.P.; Reinhorn, A.M.; Bruneau, M. Framework for analytical quantification of disaster resilience. Eng. Struct. 2010, 32, 3639-3649. [CrossRef]

30. Prasad, T.D.; Tanyimboh, T.T. Entropy based design of Anytown water distribution network. Water Distrib. Syst. Anal. 2008, 2008, 1-12.

31. Tscheikner-Gratl, F.; Egger, P.; Rauch, W.; Kleidorfer, M. Comparison of multi-criteria decision support methods for integrated rehabilitation prioritization. Water 2017, 9, 68. [CrossRef]

32. Gheisi, A.; Shabani, S.; Naser, G. Flexibility ranking of water distribution system designs under future mechanical and hydraulic uncertainty. Procedia Eng. 2015, 119, 1202-1211. [CrossRef]

33. EPANET3 in Github. Available online: https://github.com/OpenWaterAnalytics/epanet-dev/blob/master/src/ epanet3.h (accessed on 15 August 2016).

34. Pachauri, R.; Meyer, L. Climate Change 2014: Synthesis Report. Fifth Assessment Report of the Intergovernmental Panel on Climate Change; IPCC: Geneva, Switzerland, 2014.

35. Kenney, D.S.; Goemans, C.; Klein, R.; Lowrey, J.; Reidy, K. Residential water demand management: lessons from Aurora, Colorado 1. J. Am. Water Resour. Assoc. 2008, 44, 192-207. [CrossRef]

36. Hoornweg, D.; Pope, K. Population predictions for the world's largest cities in the 21st century. Environ. Urban. 2017, 29, 195-216. [CrossRef]

37. OMOECC Design Guidelines for Drinking Water Systems, Ontario Ministry of the Environment and Climate Change. Available online: http://www.ontario.ca/document/design-guidelines-drinking-water-systems (accessed on 22 May 2020).

(C) 2020 by the authors. Licensee MDPI, Basel, Switzerland. This article is an open access article distributed under the terms and conditions of the Creative Commons Attribution (CC BY) license (http://creativecommons.org/licenses/by/4.0/). 